\title{
Design of a Single-Layer Microchannel for Continuous Sheathless Single- Stream Particle Inertial Focusing
}

\begin{abstract}
High-throughput, high-precision single-stream focusing of microparticles has a potentially wide range of applications in biochemical analysis and clinical diagnosis. In this work, we develop a sheathless threedimensional (3D) particle-focusing method in a single-layer microchannel. This novel microchannel consists of periodic high-aspect-ratio curved channels and straight channels. The proposed method takes advantage of both the curved channels, which induce Dean flow to promote particle migration, and straight channels, which suppress the remaining stirring effects of Dean flow to stabilize the achieved particle focusing. The 3D particle focusing is demonstrated experimentally, and the mechanism is analyzed theoretically. The effects of flow rate, particle size, and cycle number on the focusing performance were also investigated. The experimental results demonstrate that polystyrene particles with diameters of 5-20 $\mu \mathrm{m}$ can be focused into a 3D single file within seven channel cycles, with the focusing accuracy up to $98.5 \%$ and focusing rate up to 98.97\%. The focusing throughput could reach up to 105 counts/min. Furthermore, its applicability to biological cells is also demonstrated by $3 \mathrm{D}$ focusing of HeLa and melanoma cells and bovine blood cells in the proposed microchannel. The proposed sheathless passive focusing scheme, featuring a simple channel structure, small footprint ( $9 \mathrm{~mm} \times 1.2 \mathrm{~mm}$ ), compact layout, and uncomplicated fabrication procedure, holds great promise as an efficient $3 \mathrm{D}$ focusing unit for the development of next-generation on-chip flow cytometry.
\end{abstract}

\section{Disciplines}

Engineering | Science and Technology Studies

\section{Publication Details}

Zhang, Y., Zhang, J., Tang, F., Li, W. \& Wang, X. (2018). Design of a Single-Layer Microchannel for Continuous Sheathless Single-Stream Particle Inertial Focusing. Analytical Chemistry, 90 (3), 1786-1794. 


\title{
A novel designof single-layer microchannel for continuous sheathless single-stream particle inertial focusing
}

\author{
Yan Zhang $\ddagger^{1}$, Jun Zhang $\ddagger^{2}$, Fei Tang*¹, Weihua $\mathrm{Li}^{3}$, Xiaohao Wang ${ }^{1}$ \\ 1. State Key Laboratory of Precision Measurement Technology and Instruments, Department of Precision Instrument, Tsinghua University, \\ Beijing 100084, China.
}

2. School of Mechanical Engineering, Nanjing University of Science and Technology, Nanjing 210094, China

3. School of Mechanical, Materials and Mechatronic Engineering, University of Wollongong, Wollongong, NSW 2522, Australia

Corresponding Author

* Fax: +86-10-62796707. E-mail: tangf@mail.tsinghua.edu.cn

\begin{abstract}
High-throughput, high-precision single-stream focusing of microparticles has potential wide range of applications in bio-chemical analysis and clinical diagnosis. In this work, we develop a sheathless three-dimensional (3D) particle focusing method in a single-layer microchannel. This novel microchannel consists of periodic high-aspect-ratio curved channels and straight channels. The proposed method takes advantage of both the curved channels, which induce Dean flow to promote particle migration, and straight channels, which suppress the remaining stirring effects of Dean flow to stabilize the achieved particle focusing. The 3D particle focusing is demonstrated experimentally and the mechanism is analyzed theoretically. The effects of flow rate, particle size, and cycle number on the focusing performance were also investigated. The experimental results demonstrate that polystyrene particles with diameters of 5-20 $\mu \mathrm{m}$ can be focused into a 3D single file within 7 channel cycles, with the focusing accuracy up to $98.5 \%$ and focusing rate up to $98.97 \%$. The focusing throughput could reach up to $\sim 10^{5}$ counts/min. Furthermore, its applicability to biological cells is also demonstrated by 3D focusing of HeLa and melanoma cells and bovine blood cells in the proposed microchannel. The proposed sheathless passive focusing scheme, featuring a simple channel structure, small footprint ( 9 $\mathrm{mm} \times 1.2 \mathrm{~mm}$ ), compact layout and uncomplicated fabrication procedure, holds great promise as an efficient 3D focusing unit for the development of next-generation on-chip flow cytometry.
\end{abstract}

\section{INTRODUCTION}

High-throughput ordering and focusing of cells is a prerequisite for numerous biomedical diagnostic operations, such as flow cytometry, ${ }^{1}$ cell sorting, ${ }^{2}$ deformability-based phenotyping studies, ${ }^{3}$ and protein concentration analysis. ${ }^{4}$ Although conventional benchtop instruments have achieved significant successes for the above applications,the complexity, bulkiness, and high cost of commercial instruments have shifted research efforts towards the development of their microscale counterparts. Under such circumstances, microfluidic techniques, which are associated with low cost, good portability, low volume consumption of samples/reagents, fast processing speed, massively parallel processing capacity, and impressive flow control capability, ${ }^{5-8}$ have emerged with promising potential to serve as point-ofcare diagnostic tools, especially for the precise manipulation of bioparticles.

To date, microfluidic techniques have been extensively applied for single-stream particle focusing, the schemes of which can be roughly divided into two categories: sheathflow-aided focusing and sheathless focusing. ${ }^{9}$ For the sheathflow-aided category, a funnel-like channel geometry has been widely adopted, where the inner sample fluid is hydrodynamically confined by the surrounding sheath fluid into a two-dimensional (2D) band ${ }^{10,11}$ or three-dimensional (3D) single stream. ${ }^{12,13}$ Although this mechanism has been widely used, it often involves complex multi-layer 3D structures fabricated using laborious multi-step photolithography and multi-layer assembly protocols. ${ }^{14-}$ ${ }^{16}$ Additionally, the demand for the introduction and control of extra sheath fluids inevitably complicates the operation system and, even worse, greatly dilutes the biological sample for further analysis. ${ }^{17-19}$

Instead of the hydrodynamic pinching effect of sheath flows, sheathless focusing techniquesregulate transverse particle movement by employing lateral force fields. Depending on the source of force field, sheathless particle manipulation techniques can be further categorized as active and passive methods, ${ }^{20}$ respectively. Active methods exert forces onto particles via auxiliary physical fields, such as magnetic, ${ }^{21-24}$ acoustic, ${ }^{25-27}$ electric, ${ }^{28,29}$ and optical ${ }^{30}$ fields. The introduction of an externalforce field can enhance the particle focusingaccuracy and control flexibility. ${ }^{31}$ However, the generation of extra physical fields requires the integration of peripheral control devices into the system, thus greatly increasing the fabrication complexity, control difficulty, and cost in general. In addition, the throughput of active focusing approaches is relatively low and they are difficult to be adopted in large-sample-volume applications. 
Passive methods employ the intrinsic hydrodynamic forces to manipulate cell movement, such as the deterministic lateral displacement, $^{32}$ viscoelastic focusing, ${ }^{33}$ and hydrophoretic focusing. ${ }^{34}$ The operation of these techniques lies in the region of Stokes flow, where flow inertia is negligible. In contrast, the inertial microfluidic techniqueemploys fluid inertia to manipulate particles in a high-throughput manner. Unlike the Stokes flow regime with negligible fluid inertia, inertial microfluidics operates in an intermediate range between the Stokes regime and turbulent regime, where both the inertia and viscosity of the fluid are finite. ${ }^{35}$ The finite inertia causes inertial migration, which is dominated by two effects: sheargradient-induced lift forces $\left(F_{\mathrm{SG}}\right)$ and wall-effect lift forces $\left(F_{\mathrm{WL}}\right) .^{36}$ The $F_{\mathrm{SG}}$ originates from the parabolic flow velocity profile and pushes particles toward channel walls, and $F_{\mathrm{WL}}$ directs particles toward the channel centerline. ${ }^{37}$ Accordingly, extensive efforts have been dedicated to the development of high-throughput cell focusing with the proposal of various channel structures. Hur et al. ${ }^{38}$ designed a straight-channel model to realize particle focusing by simply employing the balance of inertial lift forces. Although the proposed channel design is simple, there are more than one corresponding particle equilibrium positions and spatial single-stream ordering of particles is unachievable. Meanwhile, Wang et al. ${ }^{39}$ proposed a two-stage focusing model, which could indeed achieve single-stream particle focusing. However, this model involved asymmetric segmentation of the flow field, which places a higher demand on bifurcation design to match the flow resistance of the downstream branches.

In addition to the aforementioned inertial lift forces in straight channels, another important force called the Dean drag force ${ }^{40}$ appears in the curved channel, and it works together with the inertial lift forces to further modify the particle trajectories. Park et al. ${ }^{41}$ used a series of contractionexpansion arrays to improve the focusing performance through the combination of inertial lift and Dean flow at the corners of the arrays. Meanwhile, spiral channels ${ }^{42-44}$ with a low aspect ratio (AR) have also been widely applied for particle focusing usingthe balance between the inertial lift and Dean drag forces. In addition, asymmetric curves have been developed for high-throughput single-stream particle focusing, ${ }^{45}$ which have played a crucial role in anintegrated microfluidic system for circulatingtumorcellsseparation developed by Toner's group. ${ }^{2}$ Rafeie et al. ${ }^{46}$ made a further step by adopting slanted spiral microchannels instead of traditional rectangular cross section. However, the fabrication difficulty of the trapezoidal microchannels inevitably increases. In additionto a 2D structure within a single-layer microchannel, patterning microstructures on the top or bottom surface of the microchannel will also induce secondary flow, which can be applied for particle focusing. ${ }^{47,48,49}$ Overall, inertial microfluidics has opened up a brand new perspective for effective and robust cell focusing in a high-throughput fashion. However, prior works suffer from several limitations, such as multi-stream rather than single-stream focusing ${ }^{41,50}$ or complex multi-layer structure, which increases the chip fabrication costs. ${ }^{51,52}$

Regarding the problems aforementioned, we propose and develop a high-throughput 3D single-stream particle focusing techniqueusing a single-layer microchannel with simple structures in this work, which are comprised of periodically interconnected straight channels and semicircular curves. Within each focusing cycle, secondary flow in the curved channel is used to "drive" the particles to the outer side of the curve, and the initial "outward" distribution is immediately further reinforced by the following straight-channel section, such that potential defocusing arising from the stirring effect of secondary flow in the curvature cannot accumulate in the next cycle. The 3D particle focusing performanceis demonstrated experimentally in the proposed microchannel, and the focusing mechanism is analyzed. In addition, the effects of flow rate, particle size, and period number of the channels on the focusing performance are experimentally investigated. Furthermore, successfulfocusing of mammalian cancer cell lines (HeLa cells and melanoma cells) and blood cells validates the applicability of the proposed microchannel on biological cells. Compared with previous works, our proposed microchannelsenjoy integrative ability to passively realize spatial single-stream focusing for particle of diameter range spanning from $5 \mu \mathrm{m}$ to $20 \mu \mathrm{m}$ with particle throughput reaching up to $\sim 10^{5}$ counts $/ \mathrm{min}$. The device is also qualified to rapidly process large quantities of samples (10 $\mathrm{mL}$ in $\sim 8 \mathrm{~min}$ ), which meets the growing need for large-volume, high throughput sample tests, such as flow cytometry. The focusing performance turns out to be robust and efficient, in terms of broad operable flow rate and impressive focusing rate as discussed in a later section, with no need of complex peripheral control. Additionally, the microchannels enjoy simple single-layer structure which requires no complex multilayer photolithography and assembly techniques, thus greatly reducing the cost, difficulty and production cycle of chip fabrication process. What's more, the microchannel designed in this paper features with compact layout because of the adopted serpentine periodic series manner, thus greatly enhancing the chip portability.

\section{Particle inertial migration in straight and curved channels}

The migration behaviors of particles in microfluidics primarily result from their interaction with the surrounding flow field. At a finite Reynolds number, in addition to the viscous drag force that entrains particles along streamlines, two types of inertial lift forces exist that dominate particle lateral migration across streamlines in straight channels: shear-gradient-induced lift forces $\left(F_{\mathrm{SG}}\right)$ and wall-effect lift forces $\left(F_{\mathrm{WL}}\right){ }^{36}$ The balance of the two forces results in well-defined particle equilibrium positions in the cross-section of the microchannel. Generally, within square channels, randomly distributed particles tend to be focused at four positions along the center of each channel face. A reduction of the number of equilibrium positions from four to two centered at the long walls of the channel occurs upon increasing the channel AR (channel height/channel width) to about two, which is partly due to the blunted velocity profile along the wider channel. Notably, as the flow rate further increases, the two previously unstable equilibrium positions along the short-channel faces reappear again as $F_{W L}$ becomes less dominant than $F_{\mathrm{SG}}$, leading to recovery to the four equilibrium positions. ${ }^{36,38,53-56}$

As for curved channels, the particle migration trajectory and equilibrium positions are determined by the relative magnitude of the inertial lift and Dean drag force. ${ }^{57}$ According to the quasi-quantitative formula derived by Di Carlo et al.: ${ }^{57}$

$$
\frac{F_{Z}}{F_{D}} \sim \frac{1}{\delta}\left(\frac{a}{D_{h}}\right)^{3} R_{C}^{n}, \quad(n<0)
$$

where $F_{\mathrm{Z}}$ is the inertia lift force, $F_{\mathrm{D}}$ is the Dean drag force, $a$ is the particle diameter, $D_{\mathrm{h}}$ isthe hydraulic diameter of the channel, $R_{\mathrm{C}}$ is the channel Reynolds number, $\delta$ is the curvature 
ratio. The relative magnitude relationship between the Dean drag force and inertial lift is closely related to the particle diameter and flow rate. With increasing flow rate, the Dean dragforce exerts an increasingly significant effect on the control of particle movement. Changing the relative intensity of the Dean drag force and inertial lift can produce various particle-focusing modes. ${ }^{57,58}$ It is notable that, because of the stirring effect of the Dean flow,the curved channels enable particles to reach equilibrium positions faster.

\section{Design principle}

In this work, a simple channel structure was designed for highthroughput single-stream focusing of particles. The microchannel was comprised of periodically interconnected straight channels and semicircular curved channels with high ARs, as illustrated in Fig. 1(a). Particle 3D inertial focusing takes advantages of both components as discussed below.

The dynamic transition of the particle distribution across channel cross-sections is depicted in Fig. 1(b). Initially, particles are distributed randomly at the entrance, as illustrated for section A-A in Fig. 1(b). Entering the first straight-channel section with high AR, the particles tend to be roughly aligned along the channel sides because of the inertial lift forces, as observed in section $\mathrm{B}-\mathrm{B}$. Then, the pre-aligned particle group entersthe first curved section, where the Dean flow is induced. On one hand, particles at the inner corner are pinched toward the channel midline and then pushed outwards because the Dean drag force reaches a maximum around the midline. ${ }^{59} \mathrm{On}$ the other hand, for particles on the outer side, the Dean drag force on the particles along the centerline will be balanced by the wall lift force, and only a smallportion of particles outside thecenterline follows the circulating Dean vortex and migrates inwards. Hence, within the first curved section, the overall effect is that the majority of particles are piled up near the outer side of the curve, as indicated by section $\mathrm{C}-\mathrm{C}$.

The second straight channel further alleviates the dispersity of particle groupsby collecting them toward two corresponding inertial equilibrium positions within the straight channel. The secondary flow will vanish rapidly within the straight channel, as demonstrated by the simulation plots in Fig. 1(c). The diagram in Fig. 1(d) shows the relative magnitude of secondary flow in the straight channels and that of the curved channel. Within $200 \mu \mathrm{m}$ downstream from the straight channel, therelative magnitude of secondary flow is reduced to $10 \%$; thus, the adverse mixing effect of the Dean flow on particle focusing will be greatly inhibited. After this step, the difference between the inner and outer distributions of the particles in the channel cross-section becomes even more significant, as illustrated by section D-D. Because of the folding style of the unit series, particles from the outer side of the previous curve will become the inner side of the next curve. The relatively sparse remnant particles from the outer side of the next curve will be joined by the majority of particles from the inner side, as demonstrated by the transition from sections E-E to F-F. In summary, within each curve, the particle groups are subject to continuous convergence from inner side to outer side, thus gradually enhancing the focusing effect.

Therefore, after several cycles of curved and straight channels, all the particles will eventually be aligned along the outer side of the channel, as in section G-G. Moreover, because of the mixing effect of Dean flow in curved channels, the particle group may migrate to its equilibrium position faster than the simple straight channel, which can greatly shorten the focusing channel length.

\section{EXPERIMENTAL SECTION}

\section{Device design and fabrication}

The microfluidic chips were fabricated using standard photolithography and replica molding protocols. ${ }^{60}$ Detailed protocols of channel fabricationcould be found in the Supporting Information.The measured values of height, width, and length of the fabricated straight channels was $92 \mu \mathrm{m}, 69$ $\mu \mathrm{m}$, and $8 \mathrm{~mm}$, respectively. The straight channels in the adjacent cycle were separated by $120 \mu \mathrm{m}$ and connected with semicircular channels at both ends. The footprint of the entire channel structure was $9 \mathrm{~mm} \times 1.2 \mathrm{~mm}$.

To avoid the formation of gas bubbles within the microchannel, which would adversely disturb the flow field, the microchannel walls were expected to stay hydrophilic. Unfortunately, the initially induced hydrophilicity of PDMS by oxygen plasma exposure was temporary and tended to revert to hydrophobicity rapidly. To extend the duration of hydrophilicity of the channel walls, additional surface treatment was implemented by immediately injecting polyethylene glycol into the newly bonded PDMS-glass chip to immerse the channels and heating it at $120^{\circ} \mathrm{C}$ on a hot plate for $30 \mathrm{~min}$. The remaining modification reagents were flushed away using deionized water.

\section{Preparation of microbeads and cell suspensions}

The effectiveness of single-stream focusing was verified for both monodisperse rigid polystyrene (PS) beads and cell samples. Fluorescent PS beads with diameters of 5, 7.32, 10.4, 15.45 and 20.3 mmwere purchased andrespectively diluted in Tween 20 aqueous buffer $(0.2 \% \mathrm{v} / \mathrm{v})$ to concentrations up to $\sim 10^{5}$ particles/ml.Forcell samples, B16-F10 melanoma, HeLa cells and bovine blood cells were prepared. Details of sample preparation could be found in the Supporting Information.

\section{Measurement of microparticle focusing performance}

During observation of particle distribution within microchannels, the microfluidic device was mounted onto an inverted epifluorescence microscope (OLYMPUS IX73, Japan). Lateral migration dynamics of microparticleswasrecordedby fluorescent-intensity profiles and stacked bright-field (BF) images, the acquisition methods of which are elaborated in the Supporting Information.

\section{RESULTS AND DISCUSSION}

\section{Effect of cycle number on particle 3D focusing}

To evaluate the particle focusing effect in the designed chip, we injected a green-fluorescent-particle suspension with particle diameters of $15.45 \mu \mathrm{m}$ into the microchannels at a flow rate of0.8 $\mathrm{ml} / \mathrm{min}$. Fig. 2(a) shows the particle distribution at sampled critical sites of the channel structure. Inset (1) presents a macroscopic top-down view of the particle distribution near the channel inlet region, where the BF images were obtained by stacking 50 short-exposure photos and the fluorescence pattern (FP) was recorded with an exposure time of $100 \mathrm{~ms}$. Initially, cluttered particle swarms generally appeared with a significant trend of unilateral convergence while passing through the periodic channel structures. Within 
each cycle, the particles gradually approached to one side of the channel and eventually ended up in a single file by the

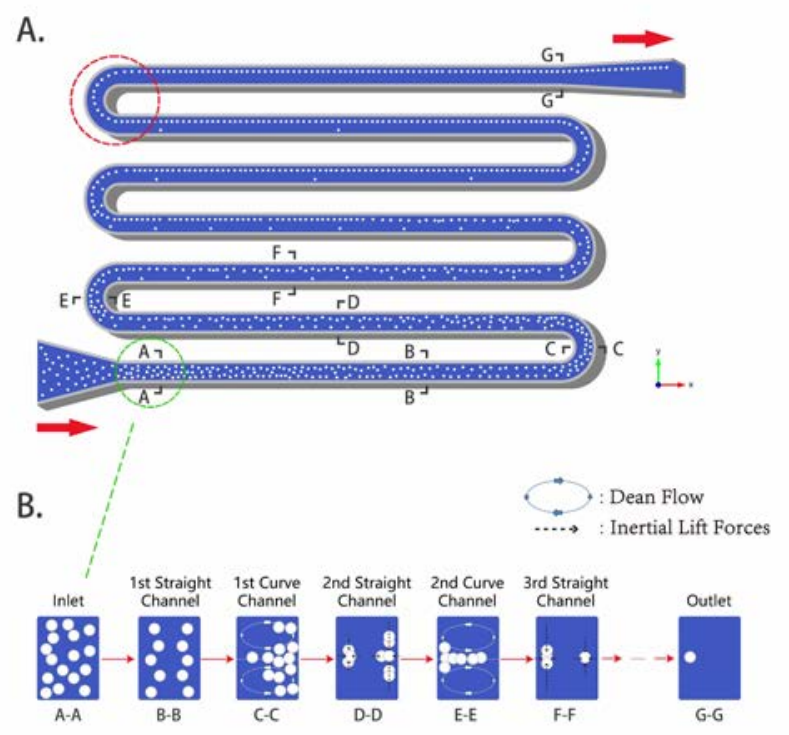

seventh cycle and advanced one by one, as indicated by gradually regular particle distribution

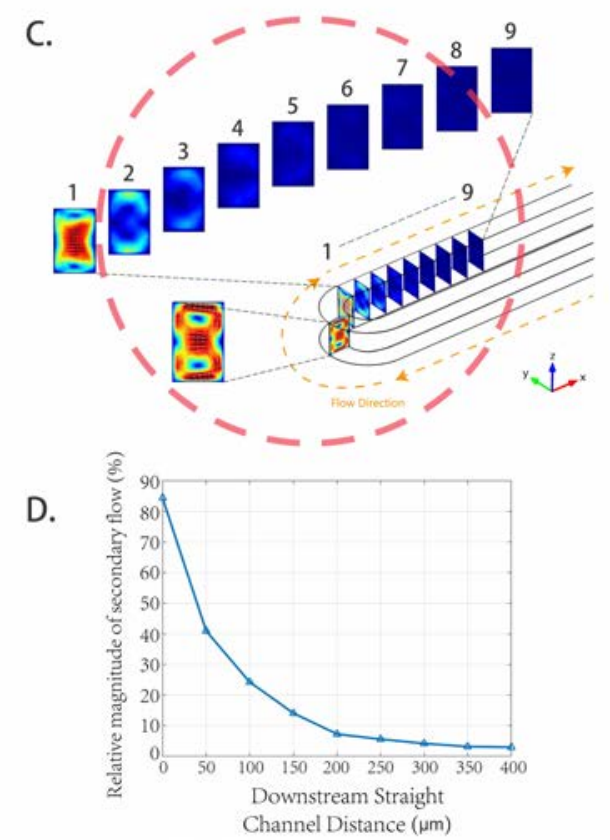

Figure 1.(A) Schematics of the 3D particle focusing using periodically interconnected straight channels and curved channels. (B) Illustration of particle migration dynamics within channel cross-section at several critical sites. (C) Progressive evolution of secondary flow fields within an adjacent curved and straight channel. Insets 1 to 9 show the secondary flow velocity ofnine consecutive cross sections along the downstream straight channel. Thedistance between eachadjacentsection is $50 \mu \mathrm{m}$. (D) Plot showing the relative magnitude ofsecondary flow in the 9 cross sections of the straight channel to that of the adjacent curved channel.

recorded in the $\mathrm{BF}$ photos and tightened fluorescence distribution in the FP images.

In addition to characterization from the top-down perspective, the particle distribution within each periodic straight channel cross-section is shown in inset (2) of Fig. 2(a), which was obtained using 3D scanning photography techniques. The particles are dispersively distributed in the channel crosssection upon entering the first cycle, as indicated by the fluorescence all over the section. Later, the cross-sectional distribution of particles shows two trends as the number of passed channel cycles increases. On one hand, the difference in the fluorescence intensity distributed on both sides of the channel becomes increasingly significant. As illustrated in inset (2), the fluorescence intensity near the left side of each section tends to diminish and eventually disappears, whereas the right-side features exhibit gradually intense fluorescence, which indicates that the majority of particles are gradually gathered to one side of the channel, which is consistent with the observations in the top-down views.

On the other hand,the distribution band of the particle swarm tends to be narrowed along the $\mathrm{z}$-axis direction. According to the recorded particle distribution across the $\mathrm{y}-\mathrm{z}$ section plane from 1 to 7 , the particles initially occupied the entire section. Afterwards, the distribution area became gradually contracted along both the z-axis and y-axis directions.Moreover, as observed since the sixth section, a central narrow area of fluorescence is present on the right side, indicating quasi-3D focusing performance. Because of the diffraction effect of light, the imaging of 3D scanning is inevitably stretchedalong the z-axis direction. Therefore, even for a perfect 3D focusing, a flat "ribbon" is observed instead of a sharp point. Inset (3) of Fig. 2(a) shows the 3D distribution of the particle swarm near the outlet. Subject to the accumulated gathering effect exerted by prior channel cycles, the particles ultimately reached the complete 3D focusing state near the theoretically expected lateral sectional position.

To quantitatively evaluate the underlying particle transition process within each cycle, fluorescent particle suspensions with particle diameters of $5,7.32,15.45$, and $20.3 \mu \mathrm{m}$ were injected into the channel at $0.8 \mathrm{ml} / \mathrm{min}$, respectively, and fluorescence patterns were recorded. Because the longexposurefluorescence intensity at one pixelis proportional to the frequency of particles passingthe corresponding position, we sampled the fluorescence intensity profile along the arrows shown in Fig. 2(b) to study the gradual tuning of the particle stream width, as illustrated in Fig. 2(c). It is evident that, regardless of the particle diameter, the particle swarms first exhibited a quasi-equilibrium state along both sides of the channel as they advanced. Specifically, according to the fluorescence intensity profile in Fig. 2(c), during the first three cycles, the fluorescence intensity pattern for the $20.3-\mu \mathrm{m}$ particles exhibited two peaks. As the particles continued flowing downstream, one of the peaksgradually disappeared, whereas the fluorescence intensity of the other rapidly increased.Therefore, a unique peak eventually remained, indicating the achievement of single-stream focusing. Accordingly, the particle-focusing transition process in the designed channel structure can be divided into two steps: rough arrangement into "two files" followed by weakening 

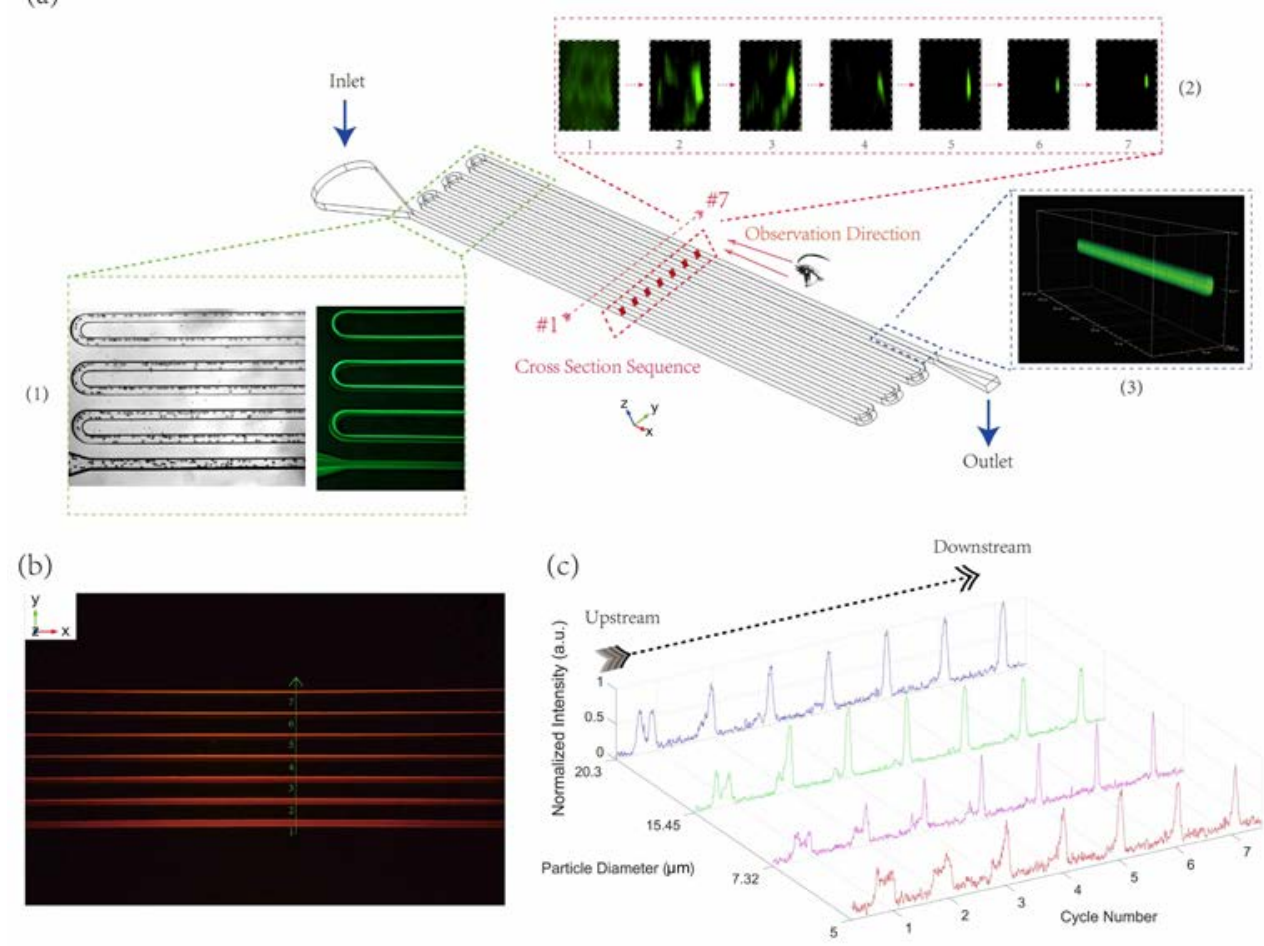

Figure 2. (a) Particle 3D inertial focusing in a microchannel consisting of periodic straight and curved channel sections (MPSC microchannel). The particle diameter is $15.45 \mu \mathrm{m}$, and the flow rate is $0.8 \mathrm{ml} / \mathrm{min}$. (1) Top-down view of particle distribution in the microchannel: (left) stacked BF image and (right) corresponding fluorescence image. (2) Confocal microscopy scanning of cross-sectional distribution of microparticles at the center of seven straight channel sections. (3) 3D trajectory of microparticles at the end of the seventh straight channel section.(b) Fluorescence image of particle distribution in straight channels; the green arrow is the sampling line for fluorescence profiles. (c) Fluorescence profiles of trajectories of differentsized microparticles (diameters of 5, 7.32, 15.45, and 20.3 $\mu \mathrm{m}$ ) sampled from seven straight cycles; the flow rate is 0.8 ml/min.

This phenomenon is due to the alternate cooperation of particle lateral migration exerted by the Dean flow in high-AR curved channel and inertial focusing effect of the high-AR straight channel. Within the high-AR straight channel, subject to inertial lift forces, the particle swarm migrates toward both sides of the channel to form a preliminary alignment. Consequently, the intensity profile exhibits two peaks of similar intensity in the first cycle of the straight channel, as observed in Fig. 2(c). In the subsequent curved channel cycles, after interaction with the Dean flow drag and inertial lift forces, the particles from the inner side of the curved channel are diverted to the outer side, resulting in a discrepancy in the bilateral distribution of the particles. The subsequent straight channel further magnifies the achieved uneven distribution of particles between both sides, and thus a fluorescence peak intensity discrepancy emerges. Benefitting from the serpentine tandem channel topology, particles flowing out of the outer side of the previous curved channel enter the inner side of the next curved channel, such that the particles continue toconverge from theinner to outer side within each cycle of curved channels, with less risks of a counteracting effect between adjacent cycles.

\section{Effect of particle diameter}

In measuring the effectiveness of particle focusing scheme, the range of focusable particle size is an important indicator that directly determines its applicability. In general, the particle size mainly affects the 1) dynamics (i.e. speed of the particle lateral migration process) and 2) steady state (i.e. particle distribution deviation after focusing).

Fig. 2(c) shows that under the given experimental conditions, the $20.3-\mu \mathrm{m}$ particles exhibit a single-stream focusing state after the fourth cycle, whereas it takes seven cycles for $5-\mu \mathrm{m}$ particles to reach a sound single-stream focusing state. The inertial migration speed of large particles ismuch faster than that of small ones, which is consistent with the prior theoretical analysis. ${ }^{59}$ It is notable that, in our tests, particles with diameters ranging from 5 to $20 \mu \mathrm{m}$ all reached an acceptable single-stream focusing state within 7 cycles. Compared with previous works employing lengthy straight channels or curvedchannels, ${ }^{34,48}$ the periodic structure of connected curved and straight channelsproposed in this work makes full use of the stirring effect of secondary flow in the curved channels and inhibition of secondary flow in the straightchannel, so as to enhancethe lateral migration of particles to the equilibrium positions, and also stabilize focusing performance of particles of a wide range of diameters, respectively.

Toquantitatively characterize the quality of particle focusing, two criteria are defined here: the (i) efficiency of the single-stream focusing state (that is, the number ratio of particles distributed into the expected single file) and (ii) accuracy of the single-stream focusing position (that is, the relative width of the focused cell stream). For a long-exposure fluorescence pattern, the fluorescence intensity profile 
represents the position distribution of all passing fluorescent particles, and thus was employed to evaluate the focusing performance. The recorded primitive fluorescence intensity profile is presented as in Fig. 2(c). To circumvent the image background noise that intervenes with qualitative analysis of the particle-focusing performance, Gaussian fitting was used for the fluorescence peak in the original intensity profile, as shown in Fig. S-1(a) of Supporting Information.

In the fluorescence profile, each pixel intensity represents the accumulated fluorescence intensity received during the exposure time, which is proportional to the number of particles passing through the corresponding position. Therefore, the relative intensity of the Gaussian peak reflects the number ratio of particles at each position, and the full-width at halfmaximum (FWHM) of the Gaussian peak characterizes the width of the focused particle stream. Accordingly, we defined two characterizing parameters: the particle focusing rate (FR) and particle focusing accuracy (FA). The focusing rate is the ratio of the Gaussian-fitted target peak height $I_{\text {target }}$ to the sum of all the Gaussian-fitted peak height values in the profile diagram, i.e., $\mathrm{FR}=\frac{I_{\text {target }}}{\sum I} \times 100 \%$. Thisparameter is used to characterize the number ratio of single-stream focused particles. The focusing accuracy is defined as $F A=(1-$ $\left.\frac{|F W H M-D|}{D}\right) \times 100 \%$, where $\mathrm{D}$ is the particle diameter, and characterizes the tightness of the single-stream alignment based on the relative difference between the focused particle distribution width and particle size.

Fig. S-1(b) summarizes the statistics of particle FR in each periodic straight channel. The FRs corresponding to all the particle sizes increased substantially and reached more than $90 \%$ within 7 cycles. In addition, the FR of larger particles increased even more sharply, with the FR of the particles with diameters of $20.3 \mu \mathrm{m}$ even reaching $98.97 \%$. This result was observed because larger particles are subject to greater inertial lift and Dean drag forces and,thus, a greater migration velocity. $^{36}$ Therefore, larger particles reach equilibrium positions much faster than smaller ones, with a higher proportion reaching the single-stream focusing state, which indicates a higher FR.

Fig. S-1(c) shows the relation between the particle FA and particle size measured near the outlet of the channel. Larger particles exhibited higher FAs, which indicates that the alignment was more strict, which is also consistent with the theoretical expectation. For particle sizes less than $5 \mu \mathrm{m}$, FA decreased abruptly. On one hand, this result occurred because the inertial migration velocity of small particles is low, such that a complete focusing state cannot be achieved in limited cycles. On the other hand, as indicated in equation (1), the adverse effect of Dean flow stirring becomes even more intense for decreased particle size, thereby further interfering with particle focusing and resulting in a wider particle stream. However, the FA of the 20.3- $\mu$ m-diameter particles was slightly lower than that of the $15.45-\mu \mathrm{m}$-diameter particles, which may be due tothe large diameter deviationfrom nominal values, the interference of which is particularly significant, within the FA range near $100 \%$.

\section{Effect of flow rate}

Based on the analysis above, in the curved channels, the induced secondary flow causes the difference in the distribution density of particles between both sides of the channel, and the periodic series connection of the curved channels continuously enhances this difference and finally results in focusing of all the particles along one side of the channel. Therefore, the key to achieving single-stream focusing is the dynamicmigration of particles in the curved channel. As a prerequisite, to achieve single-stream particle focusing, the Dean flow in the curved channel should be strong enough to drive inner particles migrating outwards, and, in turn, facilitate the convergence of two particle streams into one.The migration of particles in the curved channel is primarily determined by the relative magnitudes of the inertial lift force and Dean drag force, as discussed above. The particles will only migrate to the outer side and convergence of the inner and outer particles will only occur when the magnitude of $F_{\mathrm{D}}$ is greater than that of $F_{\mathrm{Z}}$. According to equation (1), a higher flow rate results in a more dominant effect of $F_{D}$ on the particles. Hence, if the flow rate is below a certain threshold, $F_{\mathrm{D}}$ will be insufficient to drive the particles to migrate outwards; thus, the effect of the single-stream focusing mode would be compromised and the probability of double particle streams would increase. Therefore, there is alower threshold for the flow rate, below which single-stream focusing becomes multi-stream focusing.However, there is also an upper flow rate threshold, above which the original two equilibrium positions in the high-AR straight channel tend to convert into four equilibrium positions located at the center of four channel walls, ${ }^{36}$ and thereby the single-stream focusing will also be perturbed in the proposed microchannel.

To verify theanalysisabove, a 15 - $\mu$ m-diameterfluorescent particle suspension was infused into the microchannel at flow rates from 0.2 to $1.5 \mathrm{ml} / \mathrm{min}$. The fluorescence profile at the end of the straight channel in the last cycle was recorded to characterize the particle focusing quality, and the results are presented in Fig. 3(a). At the flow rate of $0.2 \mathrm{ml} / \mathrm{min}$, there were two obvious fluorescent peaks in the channel because the Dean flow in the curved channel was not sufficiently strong and thus most of the particles were retained by the inertial lift forcesto the two equilibrium positions. When the flow rate was between 0.4 and $0.8 \mathrm{ml} / \mathrm{min}$, only one fluorescence peak appeared in the channel because the Dean flow started to play a more dominant role, driving the inner particles outward to form a single-stream focusing. An additional fluorescence peak would occasionally appear at the flow rate of $0.8 \mathrm{ml} / \mathrm{min}$, which may have arisen because the current flow rate is near the critical value, and instability of the equilibrium position starts to appear. When the flow rate was $1 \mathrm{ml} / \mathrm{min}$ or above, an additional fluorescence peak appeared in the middle of the channel, indicating that an extra equilibrium position emerged in the proposed channel. Furthermore, with increasing flow rate, the additional equilibrium position gradually stabilized, indicated by thestrengthening of the additional fluorescence peak intensity.

In addition, 3D scanning imagesof the particle trajectoryin the straight channel at the outlet, as in Fig. 3(b),further support the theoretical analysis. The particles were concentratedat both sides of the channel at $0.2 \mathrm{ml} / \mathrm{min}$. When the flow rate was increased to $0.6 \mathrm{ml} / \mathrm{min}$, complete 3D single-stream focusing was achieved. As the flow rate further increased to $1 \mathrm{ml} / \mathrm{min}$, a faint fluorescence trajectory appeared near the upper center of the channel cross-section. The intensity of the additional fluorescence streak was further enhanced when the flow rate was increased to $1.2 \mathrm{ml} / \mathrm{min}$, which is consistent with the results in Fig. 3(a). In conclusion, there is an optimal flow 
raterangewithin which $3 \mathrm{D}$ particle single-stream focusing can be achieved, as demonstrated in Fig. 3(c). As for flow rates above or below this range, the focusing rate is impaired by weakening Dean flow or occurrence of additional equilibrium positions in the straight channel, which is consistent with the theoretical analysis. According to the concentration of the prepared particle

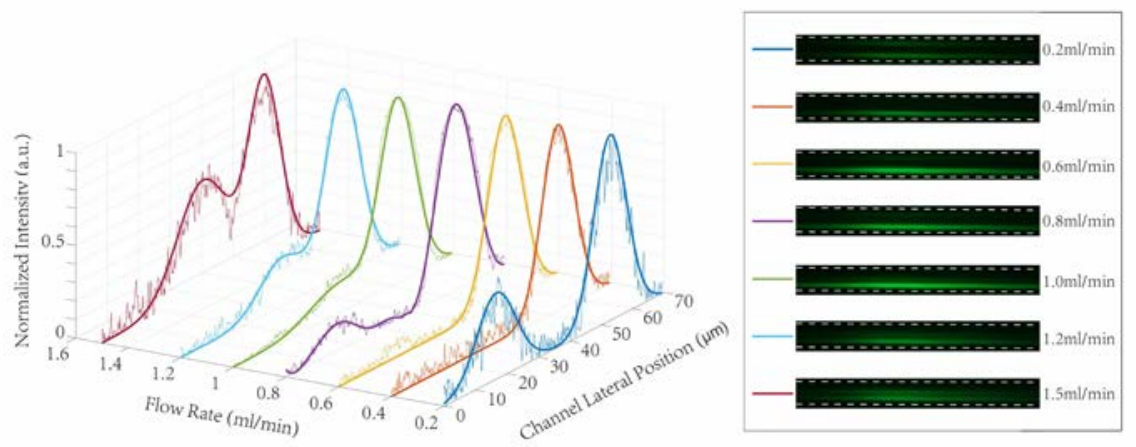

(a)

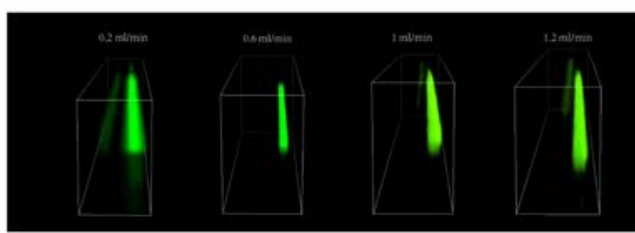

(b)

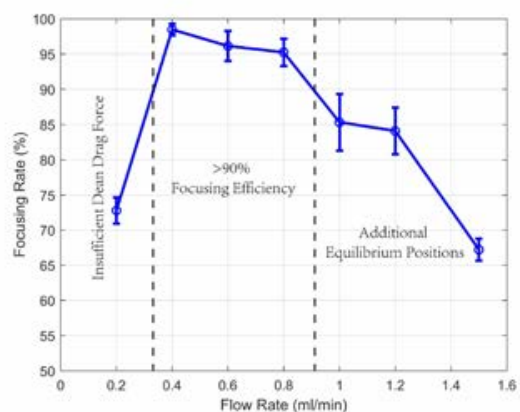

(c)

Figure 3. Effects of the flow rate on the inertial focusing performance of particles in the designed microchannel. (a) Fluorescence profiles of microparticles with flow rates of $0.2-1.5 \mathrm{ml} / \mathrm{min}$. (b) 3D trajectories of microparticles obtained from confocal microscopic scanning at the flow rates of $0.2,0.6,1$, and $1.2 \mathrm{ml} / \mathrm{min}$, respectively. (c) Three focusing regimes present within flow rate rangeof $0.2-1.5 \mathrm{ml} / \mathrm{min}$; particle diameteris $15 \mu \mathrm{m}$.

suspension and flow rate, the calculated particle throughput could reach up to $\sim 10^{5}$ counts/min in the experiment.

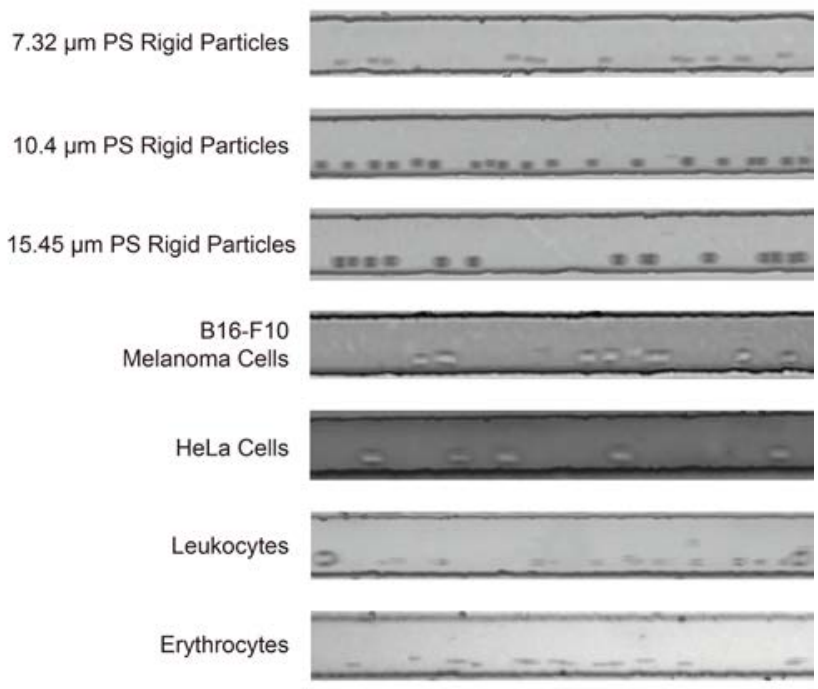

Figure 4. Inertial focusing of PS rigid particles (diameters of 7.32, 10.4, and $15.45 \mu \mathrm{m}$ ), mammalian cells (B6-F10 melanoma and HeLa cells)and blood cells (erythrocytes and leukocytes)

\section{Validation of inertial focusing for cells}

Unlike rigid PS spheres, theshape of cellular samples is imperfectly spherical because of the cell deformability, whichmay affect the inertial migration process. ${ }^{54,60}$ In this work, we have demonstrated that the proposed channel structure can also effectively achieve single-stream focusing for cell samples. Bovine blood cells (i.e. erythrocytes and leukocytes), B6-F10 melanoma and HeLa cell sample flows were injected into the channel at a flow rate of $0.8 \mathrm{ml} / \mathrm{min}$ to compare their focusing performance with those of PS beads with various diameters. Fig. 4 clearly demonstrates that the blood cells, cancercells and rigid PS particleswere respectively focused and advanced along a single file in the microchannel.It is notable that the cellular deformability and irregular shape have negligible effects on the inertial focusingin the proposed microchannel, which may be explainedby the fact that thestrength of additional lateral liftforces induced by cellular shape and deformability ${ }^{54}$ is inferior to that of dominant inertial lift forces $\left(F_{\mathrm{SG}}\right.$ and $\left.F_{\mathrm{WL}}\right)$ and secondary flow drag forces. These results validate the applicability of the proposed microchannel design for cellular sample focusing.

\section{CONCLUSION}

In this study, a novelmicrofluidic channel consisting of periodically connected straight and curved channels was proposed for particle/cell single-stream focusing. Benefitting from the stirring effect of Dean flow to driveparticles to the outer side of curve channel, and straight channels suppressing the remaining stirring effects to stabilize the achieved particle focusing, 3D focusing of particleswas efficiently realized. The experimental results demonstrated that $3 \mathrm{D}$ particle focusing 
could be achieved for particle diameters ranging from 5 to 20 $\mu \mathrm{m}$ within 7 cycles. Moreover,the focusing effect improved with increasing particle size, with a focusing accuracy up to $98.5 \%$ and focusing rate up to $98.97 \%$, with particle throughput reaching up to $\sim 10^{5}$ counts $/ \mathrm{min}$. In addition, 3D focusing performance was also verified regarding B16-F10 and HeLacancer cell and bovine blood samples. The proposed microchanneldesign enjoys a simple structure, easy fabrication, and no need for complex auxiliary control, and is promising as an efficient 3D particle focusing unit forsheathless flow cytometry of next-generation.

\section{ASSOCIATED CONTENT}

\section{Supporting Information}

Supplementary details of experimental section; Intensity profile processing and subsequent statistics of focusing performance.

\section{AUTHOR INFORMATION}

\section{Corresponding Author}

* Fax: +86-10-62796707. Tel: +86-10-62796216. E-mail: tangf@mail.tsinghua.edu.cn;

\section{Present Addresses}

† Department of Electrical and Computer Engineering, University of Illinois at Urbana-Champaign, 306 N. Wright St., Urbana, IL 61801-2918, USA

\section{Author Contributions}

All authors have given approval to the final version of the manuscript. / $¥$ These authors contributed equally.

\section{ACKNOWLEDGMENTS}

Authors sincerely thank SLSTU-NIKON Biological Imaging Center at Tsinghua University for the use of laser confocal microscope system. This work is financially supported by the National Natural Science Foundation of China (51575312), the Fundamental Research Project of Shenzhen (JCYJ20150601165744636) and the Natural Science Foundation of Jiangsu Province (Grant No. BK20170839).

\section{REFERENCES}

(1) Huh, D.; Gu, W.; Kamotani, Y.; Grotberg, J. B.; Takayama, S. Physiol Meas2005, 26, R73-R98.

(2) Ozkumur, E.; Shah, A. M.; Ciciliano, J. C.; Emmink, B. L.; Miyamoto, D. T.; Brachtel, E.; Yu, M.; Chen, P.-i.; Morgan, B.; Trautwein, J.; Kimura, A.; Sengupta, S.; Stott, S. L.; Karabacak, N. M.; Barber, T. A.; Walsh, J. R.; Smith, K.; Spuhler, P. S.; Sullivan, J. P.; Lee, R. J., et al. Science Translational Medicine2013, 5, 179ra147179 ra147.

(3) Otto, O.; Rosendahl, P.; Mietke, A.; Golfier, S.; Herold, C.; Klaue, D.; Girardo, S.; Pagliara, S.; Ekpenyong, A.; Jacobi, A.; Wobus, M.; Topfner, N.; Keyser, U. F.; Mansfeld, J.; Fischer-Friedrich, E.; Guck, J. Nat Meth2015, 12, 199-202.

(4) Hong, S. M.; Tsou, P. H.; Chou, C. K.; Yamaguchi, H.; Su, C. B.; Hung, M. C.; Kameoka, J. Biomicrofluidics2012, 6.
(5) Lecault, V.; White, A. K.; Singhal, A.; Hansen, C. L. Current Opinion in Chemical Biology2012, 16, 381390.

(6) Avesar, J.; Ben Arye, T.; Levenberg, S. Lab on a Chip2014, 14, 2161-2167.

(7) Tabeling, P. Current Opinion in Biotechnology2014, 25, 129-134.

(8) Lee, A. Lab on a Chip2013, 13, 1660-1661.

(9) Xuan, X. C.; Zhu, J. J.; Church, C. Microfluidics and Nanofluidics2010, 9, 1-16.

(10) Pamme, N.; Koyama, R.; Manz, A. Lab on a Chip2003, 3, 187-192.

(11) Che-Hsin, L.; Gwo-Bin, L. Journal of Micromechanics and Microengineering2003, 13, 447.

(12) Tsai, C.-H.; Hou, H.-H.; Fu, L.-M. Microfluidics and Nanofluidics2008, 5, 827-836.

(13) Yang, R.; Feeback, D. L.; Wang, W. Sensors and Actuators A: Physical2005, 118, 259-267.

(14) Chang, C. C.; Huang, Z. X.; Yang, R. J. Journal of Micromechanics and Microengineering2007, 17, 1479-1486.

(15) Paie, P.; Bragheri, F.; Vazquez, R. M.; Osellame, R. Lab on a Chip2014, 14, 1826-1833.

(16) Chiu, Y.-J.; Cho, S. H.; Mei, Z.; Lien, V.; Wu, T.-F.; Lo, Y.-H. Lab on a Chip2013, 13, 1803-1809.

(17) Kim, D. S.; Kim, D. S.; Han, K.; Yang, W. Microelectronic Engineering2009, 86, 1343-1346.

(18) Watkins, N.; Venkatesan, B. M.; Toner, M.; Rodriguez, W.; Bashir, R. Lab on a Chip2009, 9, 31773184.

(19) Golden, J. P.; Kim, J. S.; Erickson, J. S.; Hilliard, L. R.; Howell, P. B.; Anderson, G. P.; Nasir, M.; Ligler, F. S. Lab on a Chip2009, 9, 1942-1950.

(20) Sajeesh, P.; Sen, A. K. Microfluidics and Nanofluidics2014, 17, 1-52.

(21) Hoshino, K.; Huang, Y.-Y.; Lane, N.; Huebschman, M.; Uhr, J. W.; Frenkel, E. P.; Zhang, X. Lab on $a$ Chip2011, 11, 3449-3457.

(22) Liang, L.; Xuan, X. Microfluidics and Nanofluidics2012, 13, 637-643.

(23) Zhu, T.; Cheng, R.; Mao, L. Microfluidics and Nanofluidics2011, 11, 695-701.

(24) Jian, Z.; Chen, C.; Pallavi, V.; Vincent, B.; TzuenRong, J. T.; Xiangchun, X. Journal of Micromechanics and Microengineering2012, 22, 105018.

(25) Chen, Y.; Nawaz, A. A.; Zhao, Y.; Huang, P.-H.; McCoy, J. P.; Levine, S. J.; Wang, L.; Huang, T. J. Lab on a Chip2014, 14, 916-923.

(26) Ding, X.; Li, P.; Lin, S.-C. S.; Stratton, Z. S.; Nama, N.; Guo, F.; Slotcavage, D.; Mao, X.; Shi, J.; Costanzo, F.; Huang, T. J. Lab on a Chip2013, 13, 3626-3649. 
(27) Grenvall, C.; Antfolk, C.; Bisgaard, C. Z.; Laurell, T. Lab on a Chip2014, 14, 4629-4637.

(28) Liang, L.; Ai, Y.; Zhu, J.; Qian, S.; Xuan, X. Journal of Colloid and Interface Science2010, 347, 142-146.

(29) Gao, J.; Riahi, R.; Sin, M. L. Y.; Zhang, S.; Wong, P. K. Analyst2012, 137, 5215-5221.

(30) Zhao, Y.; Fujimoto, B. S.; Jeffries, G. D. M.; Schiro, P. G.; Chiu, D. T. Optics Express2007, 15, 6167-6176.

(31) Kung, Y. C.; Huang, K. W.; Chong, W.; Chiou, P. Y. Smal/2016, 12, 4343-4348.

(32) Morton, K. J.; Loutherback, K.; Inglis, D. W.; Tsui, O. K.; Sturm, J. C.; Chou, S. Y.; Austin, R. H. P Natl Acad Sci USA2008, 105, 7434-7438.

(33) Del Giudice, F.; Romeo, G.; D'Avino, G.; Greco, F.; Netti, P. A.; Maffettone, P. L. Lab on a Chip2013, 13, 4263-4271.

(34) Choi, S.; Song, S.; Choi, C.; Park, J. K. Small2008, 4, 634-641.

(35) Zhang, J.; Yan, S.; Yuan, D.; Alici, G.; Nguyen, N.T.; Ebrahimi Warkiani, M.; Li, W. Lab on a Chip2016, 16, 10-34.

(36) Amini, H.; Lee, W.; Di Carlo, D. Lab on a Chip2014, 14, 2739-2761.

(37) Martel, J. M.; Toner, M. Annu Rev Biomed Eng2014, 16, 371-396.

(38) Hur, S. C.; Tse, H. T. K.; Di Carlo, D. Lab on a Chip2010, 10, 274-280.

(39) Wang, X.; Zandi, M.; Ho, C. C.; Kaval, N.; Papautsky, I. Lab on a Chip2015, 15, 1812-1821.

(40) Bhagat, A. A. S.; Kuntaegowdanahalli, S. S.; Papautsky, I. Lab on a Chip2008, 8, 1906-1914.

(41) Park, J.-S.; Song, S.-H.; Jung, H.-I. Lab on a Chip2009, 9, 939-948.

(42) Bhagat, A. A. S.; Kuntaegowdanahalli, S. S.; Kaval, N.; Seliskar, C. J.; Papautsky, I. Biomedical Microdevices2010, 12, 187-195.

(43) Kuntaegowdanahalli, S. S.; Bhagat, A. A. S.; Kumar, G.; Papautsky, I. Lab on a Chip2009, 9, 29732980.

(44) Guan, G.; Wu, L.; Bhagat, A. A.; Li, Z.; Chen, P. C. Y.; Chao, S.; Ong, C. J.; Han, J. Scientific Reports2013, 3, 1475.

(45) Di Carlo, D.; Irimia, D.; Tompkins, R. G.; Toner, M. Proc Natl Acad Sci U S A2007, 104, 18892-18897.

(46) Rafeie, M.; Zhang, J.; Asadnia, M.; Li, W.; Warkiani, M. E. Lab on a Chip2016, 16, 2791-2802.

(47) Li, M.; Munoz, H. E.; Schmidt, A.; Guo, B.; Lei, C.; Goda, K.; Di Carlo, D. Lab on a Chip2016, 16, 44584465.

(48) Chung, A. J.; Gossett, D. R.; Di Carlo, D. Smal/2013, 9, 685-690.
(49) Zhao, Q.; Zhang, J.; Yan, S.; Yuan, D.; Du, H.; Alici, G.; Li, W. Scientific Reports2017, 7, 41153.

(50) Masaeli, M.; Sollier, E.; Amini, H.; Mao, W.; Camacho, K.; Doshi, N.; Mitragotri, S.; Alexeev, A.; Di Carlo, D. Physical Review X2012, 2, 031017.

(51) Chen, Y.; Chung, A. J.; Wu, T.-H.; Teitell, M. A.; Di Carlo, D.; Chiou, P.-Y. Smal/2014, 10, 1746-1751.

(52) Choi, S.; Song, S.; Choi, C.; Park, J.-K. Smal/2008, 4, 634-641.

(53) Dhar, M.; Wong, J.; Karimi, A.; Che, J.; Renier, C.; Matsumoto, M.; Triboulet, M.; Garon, E. B.; Goldman, J. W.; Rettig, M. B.; Jeffrey, S. S.; Kulkarni, R. P.; Sollier, E.; Di Carlo, D. Biomicrofluidics2015, 9, 064116.

(54) Hur, S. C.; Henderson-MacLennan, N. K.; McCabe, E. R. B.; Di Carlo, D. Lab on a Chip2011, 11, 912-920.

(55) Sollier, E.; Go, D. E.; Che, J.; Gossett, D. R.; O'Byrne, S.; Weaver, W. M.; Kummer, N.; Rettig, M.; Goldman, J.; Nickols, N.; McCloskey, S.; Kulkarni, R. P.; Di Carlo, D. Lab on a Chip2014, 14, 63-77.

(56) Gossett, D. R.; Tse, H. T. K.; Dudani, J. S.; Goda, K.; Woods, T. A.; Graves, S. W.; Di Carlo, D. Smal/2012, 8, 2757-2764.

(57) Di Carlo, D.; Irimia, D.; Tompkins, R. G.; Toner, M. P Natl Acad Sci USA2007, 104, 18892-18897.

(58) Zhang, J.; Yan, S.; Sluyter, R.; Li, W.; Alici, G.; Nguyen, N. T. Sci Rep2014, 4, 4527.

(59) Di Carlo, D. Lab on a Chip2009, 9, 3038-3046. (60) Zhou, J.; Kasper, S.; Papautsky, I. Microfluidics and Nanofluidics2013, 15, 611-623. 


\section{ForTable of Contentsonly}

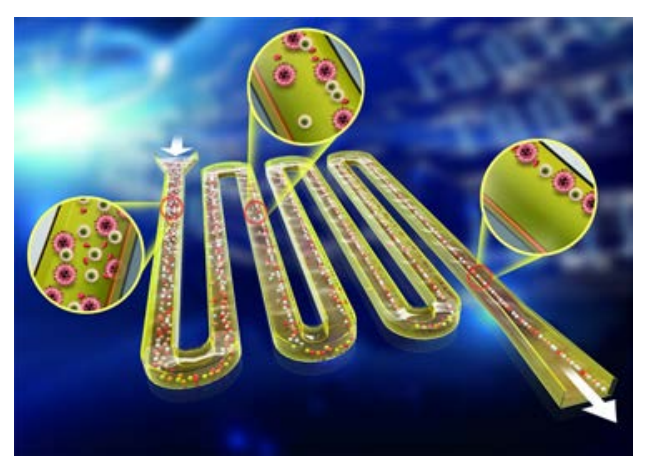

